\title{
Baculovirus expression and potential diagnostic application of the gp51 envelope glycoprotein of genetic mutants of the bovine leukaemia virus
}

\author{
Marzena Rola-Łuszczak, Agnieszka Grabowska ${ }^{1}$, \\ Bogusław Szewczyk ${ }^{1}$, Jacek Kuźmak \\ Department of Biochemistry, National Veterinary Research Institute, 24-100 Puławy, Poland \\ ${ }^{1}$ Laboratory for Recombinant Vaccines, Intercollegiate Faculty of Biotechnology, \\ University of Gdańsk and Medical University of Gdańsk, 80-309 Gdańsk, Poland \\ mrolka@piwet.pulawy.pl
}

Received: February 4, 2019 Accepted: March 1, 2019

\begin{abstract}
Introduction: Field isolates of bovine leukaemia virus (BLV) show the presence of a few amino acid substitutions in major conformational $\mathrm{G}$ and $\mathrm{H}$ epitopes on surface glycoprotein gp51. Potentially, these substitutions can affect the 3D structure of these epitopes leading to their diminished immunoreactivity. The aim of this study was to express three gp51 glycoproteins carrying mutated epitopes as recombinant baculovirus proteins in insect cells to test their immunoreactivity with bovine sera. Material and Methods: Env gene chimeras encoding mutated epitopes $\mathrm{G}$ and $\mathrm{H}$ in the env backbone of BLV FLK strain were constructed, cloned into pFastBac1 vector, and expressed in baculovirus. Results: The presence of recombinant gp51 protein in Sf9 insect cells was confirmed using monoclonal antibodies. ELISA tests were developed to check the immunoreactivity of recombinant protein with bovine sera. Conclusion: Recombinant gp51 proteins with altered G and H epitopes can be used for further studies to analyse the serological response of bovine sera towards BLV antigenic variants.
\end{abstract}

Keywords: bovine leukaemia virus, conformational epitopes, recombinant baculovirus protein.

\section{Introduction}

Bovine leukaemia virus (BLV) is a member of the Retroviridae family belonging to the Deltaretrovirus genus. BLV is recognised as the aetiologic agent for enzootic bovine leukosis, a disease that results in significant economic losses in the cattle industry worldwide. BLV infection remains asymptomatic in the large majority of infected animals. Only approximately one third of BLV-infected cattle develop lymphocytosis, while in a minority of cases (approximately 5\%), BLV infection leads to a lymphoma stage characterised by clonal expansion of B lymphocytes. Similarly to other complex retroviruses, the BLV genome contains the structural genes gag, pol, and env, regulatory genes tax, and rex, and accessory genes $R 3$ and $G 4$ (7).

Over the years, many studies on BLV genetic variability and molecular epidemiology have primarily and particularly focused on the part of the env gene encoding surface glycoprotein gp51 because of its biological functions. The extracellular gp51 protein plays a key role in the viral lifecycle, determining viral infectivity. It contains the receptor binding domain (RBD), which is indispensable for viral entry into host cells $(8,9)$. In addition, the localisation of gp51 glycoprotein at the surface of viral particles determines its role as a natural target of neutralising antibodies. Indeed, studies with different monoclonal antibodies revealed that the N-terminal part of BLV gp51 contains three conformational epitopes, F, G, and $\mathrm{H}$, which play an important role in eliciting neutralising antibodies and syncytium formation $(2,3,14)$. This was confirmed by Forti et al. (6) who showed that conformational epitopes $\mathrm{F}, \mathrm{G}$, and $\mathrm{H}$ are located in the first 173 amino acids of the N-terminal portion of gp51 protein. Further studies with monoclonal antibodies revealed the existence among BLV isolates of different origins of antigenic variants characterised by different 
expression of epitopes F, G, and H (4). In extension of this study, Mamoun et al. (11) identified the divergence at nucleotide and amino acid level among seven BLV strains and identified particular amino acids that contributed to the antigenic changes of conformational epitopes. This data was further extended over many studies with BLV isolates from different geographic origins, showing the presence of some amino acid substitutions, mainly within epitopes $\mathrm{G}$ and $\mathrm{H}(10,12$, $13,15,17)$. Potentially, these substitutions can affect the three-dimensional structure of epitopes or glycosylation of gp51 protein, leading to diminished immunoreactivity (11).

Our previous study identified the BLV isolates showing nucleotide variation in a part of the env gene encoding epitopes $\mathrm{G}$ and $\mathrm{H}$ in cattle infected with BLV (1). These mutations caused a significant amino acid variation leading to the following phenotypes: $\left(\mathrm{F}+\mathrm{G}-\mathrm{H}-\mathrm{GG}-\mathrm{G}^{-}\right), \quad\left(\mathrm{F}+\mathrm{G}+\mathrm{H}-\mathrm{GG}-\mathrm{G}^{-}\right)$, and $(\mathrm{F}+\mathrm{G}-\mathrm{H}+\mathrm{GG}-\mathrm{G}-)$ in the isolates from cattle \#317, $\# 306$, and \#18, respectively. Furthermore, for these animals, some discrepancy between the results of the PCR and the serological test was observed, which implies that inactivation of the epitope may diminish antigenicity and complicate diagnosis. In this paper, we constructed env gene chimeras encoding mutated epitopes $\mathrm{G}$ and $\mathrm{H}$ in the env backbone of the foetal lamb kidney (FLK) strain of BLV. Next, these chimeras were expressed as recombinant baculovirus proteins in insect cells and recombinant gp51 proteins were tested for their immunoreactivity with bovine sera with the aim of using them as potential diagnostic reagents.

\section{Material and Methods}

Construction of plasmids containing epitopespecific gp51 sequences. The gp51 gene was amplified from genomic DNA extracted from BLV-infected FLK cells with primers containing the PstI restriction site and START (5'-GCTGCAGGCCTTCAAATGCCT AAA-3') and STOP codon (UAA) recognition site (5'-CGACTTAACTACGTCTGACCC-3'). Then, the amplification product was cloned into pCRBlunt plasmid (Qiagen, USA), and an FLK-ATG-gp51pCRBlunt plasmid was constructed. Plasmids carrying $286 \mathrm{bp}$ fragments of the env gene encoding respective amino acids of epitopes $\mathrm{G}, \mathrm{H}, \mathrm{GG}$, and $\mathrm{G}$ were constructed by nested PCR amplification of genomic DNA from peripheral blood mononuclear cells (PBMC) of cattle \#317, \#306, and \#18 from the previous investigation (1) (Table 1), using two sets of primers (5'-CCTGGCGTTTGCTGAAAGCCTT-3' with 5'-AA ACCGGCGCCGCCCTTGTGGG-3' and 5'-CGACGG TCCCGAAGACGCCC-3' with 5'-AACAACAACCTC TGGGAAGG-3'), and subsequently cloned into pDRIVE plasmid (Qiagen, USA). Next, these plasmids were used to amplify a $146 \mathrm{bp}$ fragment with primers containing the AvrII (forward) and BglII (reverse) restriction sites (5'-CTCCCTGTCCCTAGGAAATC3' and 5'-GGGGGCCAATAGATCTTAGG-3'). After digestion with $A v r \mathrm{II} / B g l \mathrm{II}$ restriction enzymes, the fragments were ligated substituting the corresponding fragment of FLK-ATG-gp51/pCR Blunt plasmid, and as a result, recombinant plasmids containing epitope-specific sequences were created (FLK-ATGgp51/317/pCRBlunt, FLK-ATG-gp51/306/pCRBlunt, and FLK-ATG-gp51/18/pCRBlunt). Plasmid FLKATG-gp51-pCRBlunt was used to generate wild-type gp51. Finally, DNA sequencing of the constructed plasmids was performed to confirm that no difference existed between the inserts and the original variants or between the env backbone of BLV FLK and the parental strain.

Construction of recombinant baculovirus vectors. The gp51 gene was recovered from plasmids carrying epitope-encoding sequences after digestion with PstI and then subcloned into the baculovirus transfer vector $\mathrm{pFastBac1}$ (Invitrogen, USA), using the Pst I restriction site. The recombinant baculovirus transfer constructs pFastBac1-317, pFastBac1-306, pFastBac1-18, and pFastBac1-FLK were used for sitespecific transposition in E. coli (strain DH10Bac). Recombinant bacmids (baculovirus shuttle vector, large low-copy plasmid) containing the gp51 gene or gp51 variants were used to transfect insect cells ( $\mathrm{Sf} 9$ cell) to produce recombinant baculoviruses. Recombinant proteins gp51/FLK or gp51/FLK/317, gp51/FLK/306, or gp51/FLK/18 variants were identified by the immunoperoxidase monolayer assay (IPMA) with monoclonal antibody to the D-D' epitope of BLV (BLV2, Veterinary Medical Research and Development, USA) and 3-amino-9-ethylocarbazole (AEC, Sigma-Aldrich, Poland) as a substrate.

Expression and characterisation of recombinant gp51. The recombinant baculoviruses were used to infect Sf9 cells $\left(5 \times 10^{5}\right)$ at low MOI and cell cultures were harvested 5-7 days post infection. The harvested cells and supernatants were separated by centrifugation at $13,000 \mathrm{rpm}$ for $10 \mathrm{~min}$. Culture supernatant, membrane, and cytoplasmic fractions were examined for recombinant gp51 protein by SDS/PAGE and western blotting using monoclonal antibody to the D-D' epitope of BLV and peroxidase conjugated rabbit anti-mouse IgG (Sigma-Aldrich, Poland). Levels of expressed recombinant gp51 proteins were determined by enhanced chemiluminescence (ECL) using SuperSignal West Pico Chemiluminescent substrate (ThermoScientific, Poland).

ELISA with recombinant gp51 proteins. Four ELISA tests were performed with respective recombinant antigens. Immulux HB ELISA plates (Dynex Technologies, Germany) were coated with carbonate buffer-diluted culture medium at 1:10 collected after the fourth passage of Sf9 cells infected with recombinant baculoviruses gp51/FLK, gp51/FLK/317, gp51/FLK/306, and gp51/FLK/18. The 
plates were incubated overnight at $4^{\circ} \mathrm{C}$ and then washed three times with PBST buffer with $20 \%$ horse serum (Sigma-Aldrich, Poland). The tested sera were diluted 1:100 in PBST buffer with $1 \%$ of horse serum and were incubated in duplicate for $1 \mathrm{~h}$ at $37^{\circ} \mathrm{C}$. Then, the plates were washed five times, incubated with mouse IgG anti-bovine IgG labelled with peroxidase for $45 \mathrm{~min}$ at $37^{\circ} \mathrm{C}$ and washed again, and $100 \mu \mathrm{L}$ of $2,2^{\prime}$-azino-di(3-ethylbenzthiazoline sulfonic acid) solution was added to each well. After $15 \mathrm{~min}$ of incubation, the reaction was stopped and the optical density (OD) was measured at $405 \mathrm{~nm}$. The tested sera included the positive control serum $(\mathrm{P})$, which was prepared from five cattle naturally infected with BLV. All these animals' samples were positive in PCR and in sequence analysis of amplified $444 \mathrm{bp}$ fragments, confirming sequence conservation within epitopes $F, G$, and $H$ with respect to the BLV FLK reference strain. The negative control serum $(\mathrm{N})$ was prepared from five cattle seronegative in commercial ELISA and negative in PCR. Homologous sera were also tested from cattle designated \#317, \#306, and \#18 in our preceding study, (1) which were naturally infected with BLV isolates.

\section{Results}

The sequence analysis of the cloned env gene revealed the presence of a single mutation $\mathrm{C} \rightarrow \mathrm{T}$ at position 514 resulting in one amino acid change $(\mathrm{A} \rightarrow \mathrm{S})$ at position 172, as compared to the parental strain (BLV-FLK, GenBank accession no. M35242). However, this substitution was irrelevant for gp51 immunogenicity since it was located away from the region encoding epitopes and was frequently found in other isolates (17).

After transfection of Sf9 cells with recombinant bacmid DNAs, the monoclonal antibody to epitope DD' identified the gp51 backbone in cells infected by recombinant baculoviruses gp51/FLK, gp51/FLK/317, gp51/FLK/306, and gp51/FLK/18 but not in cells infected with wild-type baculovirus (WT) (Fig. 1). Expression of gp51 protein evaluated by SDS-PAGE in cell lysates (membrane fraction and cytoplasm) and supernatant showed for each construct a single protein band of molecular weight of 51-kDa, corresponding to the expected size of the Env protein (data not shown). In Western blotting developed by ECL, the expressed protein was reactive with a monoclonal antibody to epitope D-D' of BLV (Fig. 2). The recombinant gp51 protein band was clearly visible in the supernatant fractions collected from cells infected with FLK and three variants, suggesting that the recombinant protein was released into the medium of the infected Sf9 cells. The protein band representing the membrane fraction was also visible in the form of a "smear" typical for the glycosylation process in endoplasmic reticulum. No band of the corresponding molecular weight was observed when Sf9 cells were infected with wild-type baculovirus.
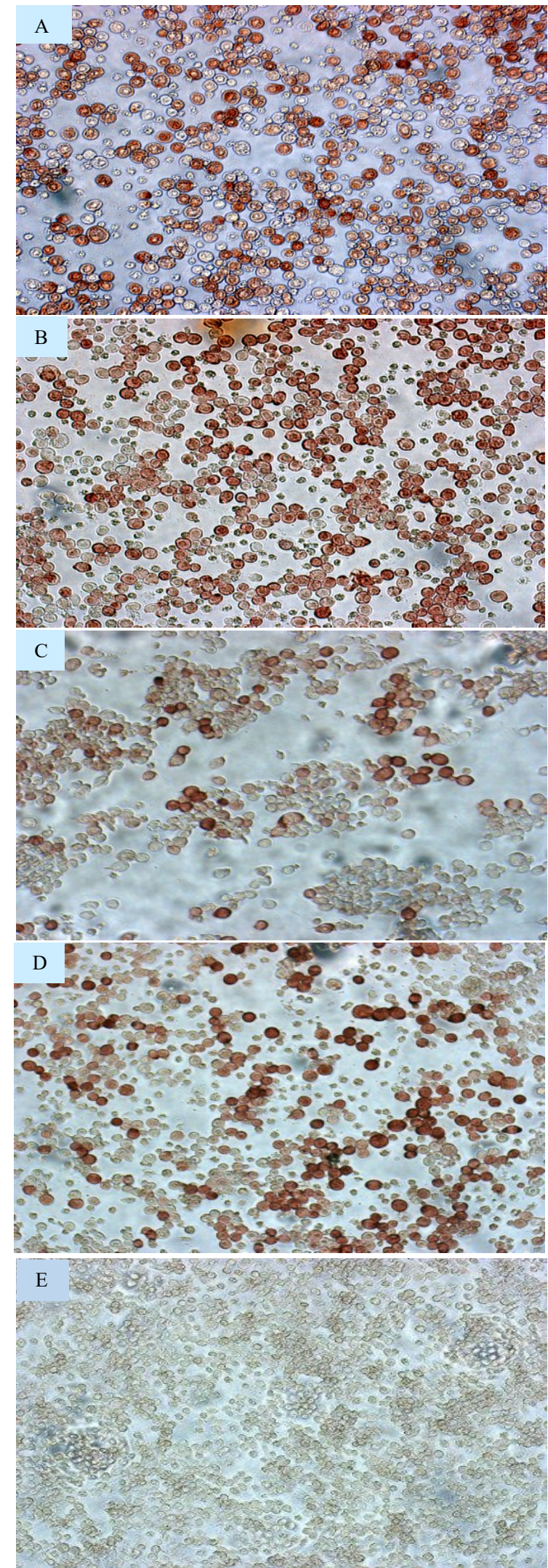

Fig. 1. Immunodetection of recombinant baculovirus gp51 proteins by IPMA in Sf9 cells. (A, B, C, D - recombinant baculoviruses gp51/FLK/18, gp51/FLK/306, gp51/FLK/317, and gp51/FLK respectively, E - cells transfected with wild type baculovirus) 
Finally, an ELISA was performed to assess the reactivity of recombinant baculovirus proteins with bovine sera. Different dilutions of supernatant fractions and of control and homologous sera were used to optimise the results. The ELISA carried out with recombinant gp51/FLK antigen reacted effectively with $\mathrm{P}$ control serum since the resulting OD value was about 2.5 times higher than with $\mathrm{N}$ control serum (Table 2). In contrast, no cross reactivity was noted between recombinant gp51/FLK antigen and homologous sera.
Similarly to gp51/FLK, high binding capacity was observed for the recombinant gp51/FLK/18 and gp51/FLK/306 antigens which reacted with respective homologous sera but not with $\mathrm{P}$ control serum. Interestingly, in ELISA, the $\mathrm{G}-$ and $\mathrm{H}$-epitope-lacking antigen gp51/FLK/317, generated from Sf9 cells infected with recombinant baculovirus, interacted poorly with homologous serum since the OD value was comparable to that noted for negative serum $\mathrm{N}$.

Table 1. Phenotypes and epitope nucleotide sequences of BLV wild type (FLK) and genetic variants used in this study

\begin{tabular}{lllll}
\hline Phenotype of epitope & Epitope G & Epitope H & Epitope GG & Epitope G \\
\hline FLK $(\mathrm{G}+\mathrm{H}+\mathrm{GG}+\mathrm{G}+)$ & GCA (Ala) & TCC (Ser) TCC (Ser) & GCC (Ala) AAG (Lys) & TCT (Ser) \\
$\# 317(\mathrm{G}-\mathrm{H}-\mathrm{GG}-\mathrm{G}-)$ & ACA (Thr) & TCC (Ser) GCC Pro (Ala) & CCC (Pro) AGG (Arg) & TTT (Phe) \\
$\# 306(\mathrm{G}+\mathrm{H}-\mathrm{GG}-\mathrm{G}-)$ & GCA (Ala) & TCC (Ser) GCC (Ala) & CCC (Pro) AGG (Arg) & TTT (Phe) \\
$\# 18(\mathrm{G}-\mathrm{H}+\mathrm{GG}-\mathrm{G}-)$ & ACA (Thr) & TCC (Ser) TCC (Ser) & CCC (Pro) AGG (Arg) & TTT (Phe) \\
\hline
\end{tabular}

Table 2. OD values of ELISA tests showing the reactivity of different recombinant antigens with serum samples

\begin{tabular}{|c|c|c|c|c|c|}
\hline \multirow{2}{*}{ Antigen } & \multicolumn{3}{|c|}{ Homologous sera } & \multirow{2}{*}{ Positive control serum P } & \multirow{2}{*}{ Negative control serum $\mathrm{N}$} \\
\hline & \#317 & \#306 & $\# 18$ & & \\
\hline gp51/FLK & 0.389 & 0.519 & 0.611 & 1.668 & 0.669 \\
\hline gp51/FLK/317 & 0.503 & 0.420 & 0.501 & 0.705 & 0.597 \\
\hline gp51/FLK/306 & 0.320 & 1.150 & 0.520 & 0.610 & 0.393 \\
\hline gp51/FLK/18 & nd & nd & 1.099 & 0.755 & 0.543 \\
\hline
\end{tabular}
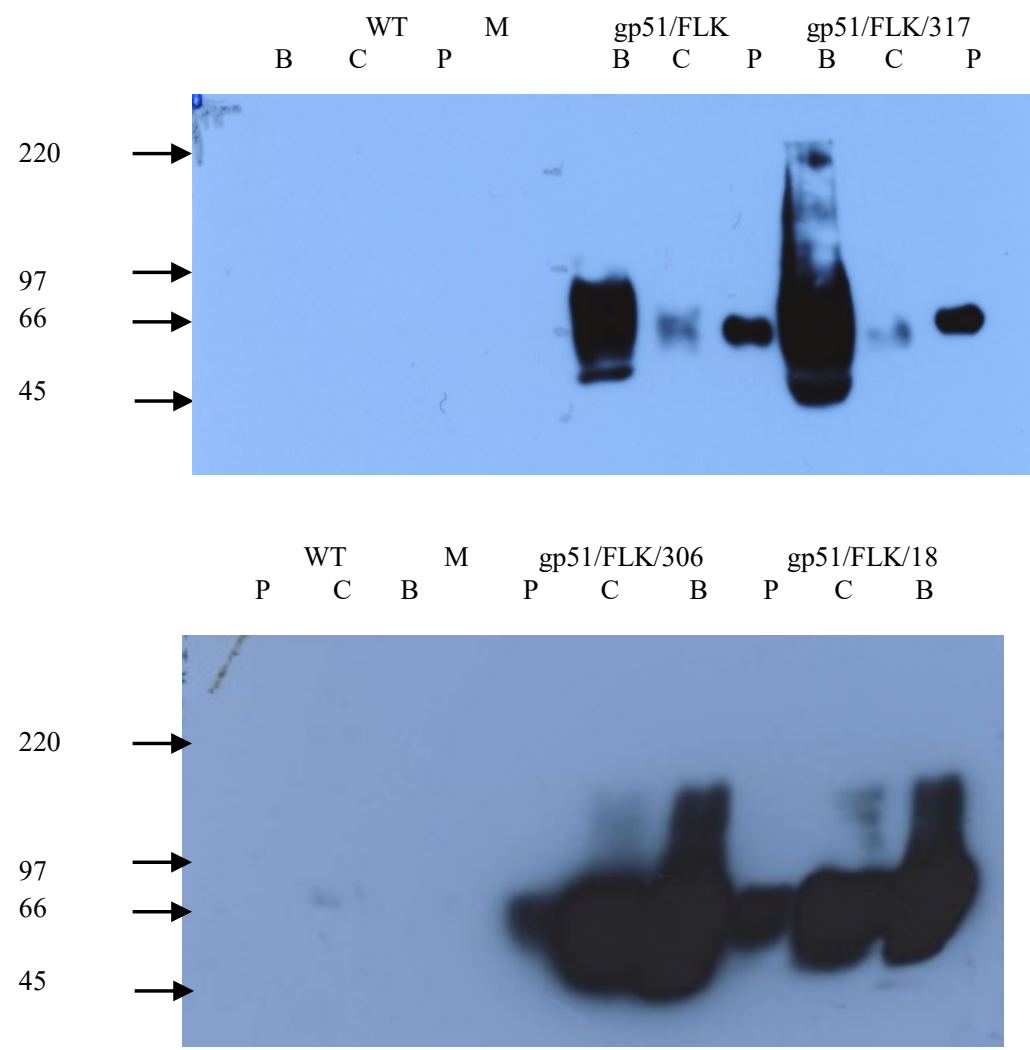

Fig. 2. Western blot-ECL analysis of recombinant baculovirus proteins. $\mathrm{B}$ - proteins in membrane fraction, $\mathrm{C}$ - proteins in cytoplasmic fraction, and $\mathrm{P}$ - proteins in culture medium. WT - cells transfected with wild type baculovirus, M - Amersham High Range Rainbow protein ladder (Sigma-Aldrich, Poland) 


\section{Discussion}

Generally, serological tests like ELISA constitute excellent tools for detection of specific antibodies to $\mathrm{BLV}$ infection. An important prerequisite for this test is the use of a diagnostic antigen capable of detecting a specific type of antibodies from infected individuals. These tests largely employ gp51 antigen prepared from FLK infected by a known isolate of BLV, with an $\mathrm{F}+\mathrm{G}+\mathrm{H}+$ phenotype for conformational epitopes (11). Alignment of the partial env region encoding the N-terminal part of gp51 revealed a number of nucleotide and amino acid substitutions that were located in functional epitopes or neutralisation domains (13). However, no serological types have been detected so far among different BLV isolates. Amino acid changes in immunoreactive epitopes raise questions about those epitopes' immunogenicity and the possible nature of strain-specific antibody response, as was shown in patients infected with antigenic mutants of HTLV type 1 (16).

In this study, recombinant baculoviruses expressing a mutated $146 \mathrm{bp}$ fragment encoding an epitope region of gp51 protein were developed. An unquestionable advantage of the cloning system used (5) was the incorporation of the signal peptide at the N-terminal part of gp51, allowing efficient transportation of the Env protein on the cell surface and its secretion to the culture medium for further use as an antigen in ELISA. ELISA data revealed that recombinant gp51/FLK/18 and gp51/FLK/306 antigens showed high binding capacity to homologous sera and reacted poorly with $\mathrm{P}$ serum, highlighting that the amino acid sequence of the epitope region presumably determines the avidity of the reaction. Indeed, the isolate \#18 lacks epitope $G$ (Table 1), which plays a critical role in BLV infectivity and immune escape (17). A high ratio of the rate of synonymous to the rate of non-synonymous substitutions ( $\mathrm{dN} / \mathrm{dS})$ observed for this epitope $(12,17)$ indicates its stringent positive selection and involvement in the evolution of escape mutants. Isolate \#306 was characterised by amino acid changes in a part of epitope $G$ and complete lack of epitope H (Table 1). In this epitope, substitution of the small polar amino acid Ser-58 with the small hydrophobic amino acid Ala-58 corresponded to a diminution of immunoreactivity (11). We cannot clarify the diminished reactivity of gp51/FLK/317 toward homologous serum. Possibly, it could be caused by an alteration of the conformational structure of gp 51 protein due to missing both $\mathrm{G}$ and $\mathrm{H}$ epitopes.

In summary, recombinant gp51 proteins which represent altered Env conformational epitopes $\mathrm{G}$ and $\mathrm{H}$ can be suitable reagents for further study to analyse infections caused by BLV variants, especially in the context of serological diagnosis. These proteins particularly commend themselves to serological investigation when blocking or competition ELISA tests employing monoclonal conjugate directed to defined epitopes are used.

Conflict of Interests Statement: The authors declare that there is no conflict of interests regarding the publication of this article.

Financial Disclosure Statement: This work was supported by the Polish Ministry of Science and Higher Education (grant no. 2 PO4B 009 27).

Animal Rights Statement: None required.

\section{References}

1. Bicka L., Rola M., Kozaczyńska B., Stec J., Kuźmak J.: Characteristics of genetic mutants of bovine leukaemia virus and their role in serodiagnosing BLV infections. Med Weter 2005, 61, 699-702.

2. Bruck C., Portetelle D., Burny A., Zavada J.: Topographical analysis by monoclonal antibodies of BLV-gp51 epitopes involved in viral functions. Virology 1982, 122, 353-362.

3. Bruck C., Mathot S., Portetelle D., Berte C., Franssen J.D., Herion P., Burny A.: Monoclonal antibodies define eight independent antigenic regions on the bovine leukemia virus (BLV) envelope glycoprotein gp51. Virology 1982, 122, 342-352.

4. Bruck C., Rensonnet N., Portetelle D., Cleuter Y., Mammerickx M., Burny A., Mamoun R., Guillemain B., Van der Maaten M.J., Ghysdael J.: Biologically active epitopes of bovine leukemia virus glycoprotein gp51: their dependence on protein glycosylation and genetic variability. Virology 1984, 136, 20-31.

5. De Giuseppe A., Feliziani F., Rutili D., De Mia G.M.: Expression of the bovine leukemia virus envelope glycoprotein (gp51) by recombinant baculovirus and its use in an enzymelinked immunosorbent assay. Clin Diagn Lab Immunol 2004, 11, 147-151.

6. Forti K., Rizzo G., Cagiola M., Ferrante G., Marini C., Feliziani F., Pezzotti G., De Giuseppe A.: Identification of a novel overlapping sequential E epitope (E') on the bovine leukaemia virus SU glycoprotein and analysis of immunological data. Vet Microbiol 2014, 172, 157-167.

7. Gillet N., Florins A., Boxus M., Burteau C., Nigro A., Vandermeers F., Balon H., Bouzar A., Defoiche J, Burny A, Reichert M, Kettmann R, Willems L.: Mechanisms of leukemogenesis induced by bovine leukemia virus: prospects for novel anti-retroviral therapies in human. Retrovirology 2007, 16, $4-18$.

8. Johnston E., Albritton L., Radke K.: Envelope proteins containing single amino acid substitutions support a structural model of the receptor - binding domain of bovine leukemia virus. J Virol 2002, 76, 10861-10872.

9. Lavanya M., Kinet S., Montel-Hagen A., Mongelaz C., Battini J.L., Taylor N.: Cell surface expression of the bovine leukemia virus-binding receptor on $\mathrm{B}$ and $\mathrm{T}$ lymphocytes is induced by receptor engagement. J Immunol 2008, 181, 891-898.

10. Lee E., Kim E.J., Ratthanophart J., Vitoonpong R., Kim B.H., Cho I.S., Song J.Y., Lee K.K, Shin Y.K.: Molecular epidemiological and serological studies of bovine leukemia virus (BLV) infection in Thailand cattle. Infect Genet Evol 2016, 41, 245-254.

11. Mamoun R.Z., Morrison M., Rebeyrotte N., Busetta B., Couez D., Kettmann R., Hospital M., Guillemain B.: Sequence variability of bovine leukemia virus env gene and its relevance to the structure and antigenicity of the glycoproteins. J Virol 1990, 64, 4180-4188. 
12. Pluta A., Rola-Łuszczak M., Kubiś P., Balov S., Moskalik R., Choudhury B., Kuźmak J.: Molecular characterization of bovine leukemia virus from Moldovan dairy cattle. Arch Virol 2017, 162, 1563-1576.

13. Polat M., Moe H.H, Shimogiri T., Moe K.K., Takeshima S.N, Aida Y.: The molecular epidemiological study of bovine leukemia virus infection in Myanmar cattle. Arch Virol 2017, $162,425-437$.

14. Portetelle D., Dandoy C., Burny A., Zavada J., Siakkou H., Geas-Masse H., Drobecq H., Tartar A.: Synthetic peptides approach to identification of epitopes on bovine leukemia virus envelope glycoprotein gp51. Virology 1989, 169, 34-41.

15. Rola-Łuszczak M., Pluta A., Olech M., Donnik I., Petropavlovskiy M., Gerilovych A., Vinogradova I., Choudhury B.,
Kuźmak J.: The molecular characterization of bovine leukaemia virus isolates from Eastern Europe and Siberia and its impact on phylogeny. PLoS One 2013, 8, e58705. Doi: 10.1371/journal. pone.0058705.

16. Tallet B., Astier-Gin T., Moynet D., Londos-Gagliardi D., Guillemain B.: Sequence variations in the amino- and carboxyterminal parts of the surface envelope glycoprotein of HTLV type 1 induce specific neutralizing antibodies. AIDS Res Human Retroviruses 2001, 17, 337-348.

17. Zhao X., Buehring G.C.: Natural genetic variations in bovine leukemia virus envelope gene: Possible effects of selection and escape. Virology 2007, 366, 150-165. 